\title{
Scleral buckling procedure with chandelier illumination for pediatric rhegmatogenous retinal detachment
}

This article was published in the following Dove Press journal:

Clinical Ophthalmology

23 January 2015

Number of times this article has been viewed

\section{Toshiyuki Yokoyama \\ Koki Kanbayashi \\ Tamaki Yamaguchi}

Department of Ophthalmology, Juntendo University Nerima Hospital, Tokyo, Japan
Correspondence: Toshiyuki Yokoyama Department of Ophthalmology, Juntendo University Nerima Hospital, 3-I-10 Takanodai, Nerima-ku, Tokyo 177-852I, Japan

Tel +81359233111

Fax +8I 359233217

Email yokoyama@juntendo-nerima.jp
Purpose: To assess the treatment of pediatric patients with rhegmatogenous retinal detachment (RRD) by scleral buckling with chandelier illumination.

Methods: Three eyes were treated in three patients, healthy boys aged 7 years, 12 years, and 11 years, with RRD, macular involvement, and small retinal holes, of which two were preoperatively undetectable. Conventional scleral buckling with cryoretinopexy was performed under the contact lens for vitreous surgery or noncontact wide-angle viewing system using 27 -gauge twin chandelier illumination.

Results: The only known predisposing factor for retinal detachment was myopia stronger than $3 \mathrm{D}$ with lattice retinal degeneration in two of the three patients. Retinal reattachment was achieved in all cases without intra- or postoperative complications. However, visual recovery was limited in one of the three patients.

Conclusion: Scleral buckling with chandelier illumination is effective for pediatric RRD, especially if the retinal hole is difficult to detect preoperatively. However, visual recovery was sometimes limited because of macular involvement due to late diagnosis, which is one of the characteristic features of pediatric RRD.

Keywords: pediatric rhegmatogenous retinal detachment, chandelier illumination, scleral buckling

\section{Introduction}

Rhegmatogenous retinal detachment (RRD) is uncommon in the pediatric population, accounting for only $1.7 \%-12.6 \%$ of all cases of RRD, and is characterized by a high incidence of macular involvement and proliferative vitreoretinopathy (PVR) at presentation due to delayed diagnosis, the presence of bilateral involvement and preoperatively undetectable retinal holes, and male predominance. ${ }^{1-11}$ Most patients have predisposing factors such as trauma, myopia, congenital or developing anomalies, uveitis, and previous intraocular surgery. Bilateral retinal detachment is often associated with pediatric RRD together with a high incidence of retinal pathologies, ${ }^{4-6,10}$ possibly because the predisposing factors such as congenital or developmental anomaly and myopia usually occur bilaterally. Surgical treatment using vitrectomy is often performed in adult RRD patients. However, the cortical vitreous is difficult to remove completely, so a scleral buckling procedure is preferred in pediatric RRD patients, except in the presence of severe PVR, opaque media, or undetectable break.

Chronic retinal detachment is the most characteristic feature of pediatric RRD due to the delay of diagnosis. Most patients have no complaints or cannot complain of decreased vision, and are incidentally identified by screening such as school vision tests. Thorough examinations such as scleral indentation to find the peripheral lesion or 
even fundus examination using the ophthalmoscope may be difficult in pediatric patients. Consequently, most detachments involve the macula, and many patients had PVR at the time of diagnosis that often results in poor functional prognosis. ${ }^{1-11}$

We treated three pediatric RRD patients, two of whom had preoperatively undetected retinal hole, using the buckling procedure under the contact lens for vitreous surgery or the noncontact wide-angle viewing system with 27-gauge twin chandelier illumination. We discuss the advantages and difficulties of treating pediatric RRD patients with this procedure.

\section{Patients and methods}

The study was approved by the institutional review board of Juntendo University Nerima Hospital. All patients and their parents gave informed consent, and this study was conducted in accordance with the Declaration of Helsinki. The visual acuity of all eyes was measured using the decimal best-corrected visual acuity.

\section{Case I}

A 7-year-old boy had decreased visual acuity in the right eye detected at a school vision-screening test and was referred to us for detailed assessment by a local ophthalmologist who suspected amblyopia in June 2012. He had a history of bronchial asthma, but no history of ocular trauma or atopic dermatitis, and had no relevant family history. At his first visit, the best-corrected visual acuity was 0.3 with slight hyperopic astigmatism in the right eye. Slit-lamp examination revealed only slight superficial punctate keratopathy in the right eye. Fundus examination revealed flat peripheral retinal detachment extending from 4 to 10 o'clock with some subretinal strands in the right eye, but no retinal break was detected despite careful examinations. Although the macula was attached, optical coherence tomography (OCT) revealed a central-to-inferior defect of the inner and outer segment junction line of the photoreceptor, probably indicating previous macular detachment (Figure 1). No abnormality was detected in his left eye with visual acuity of 1.2.

\section{Case 2}

A 12-year-old boy had decreased visual acuity in his left eye detected at examination for contact lenses by a local ophthalmologist, and was referred to us for more detailed examinations in September 2012. He had no remarkable past or family history except hay fever, and had no history of ocular trauma or atopic dermatitis. His best-corrected visual acuity was 1.2 with myopia of $-4.00 \mathrm{D}$ in the right eye, and 0.2 with myopia of $-3.50 \mathrm{D}$ in the left eye. Slit-lamp examination revealed no abnormalities in both eyes. Fundus examination revealed flat retinal detachment extending from 3 to 9 o'clock involving the macula with lattice degenerations at 5 and 7 o'clock and subretinal strands in the left eye (Figure 2). However, no retinal break was detected. Lattice degeneration was found with a hole in the peripheral temporal retina in the right eye.

\section{Case 3}

An 11-year-old boy visited a local ophthalmic clinic complaining of blurred vision in the right eye, and was referred to us under a diagnosis of decreased vision with unknown cause in October 2012. His best-corrected visual acuity was 0.2 with myopia of $-5.75 \mathrm{D}$ in the right eye and 1.2

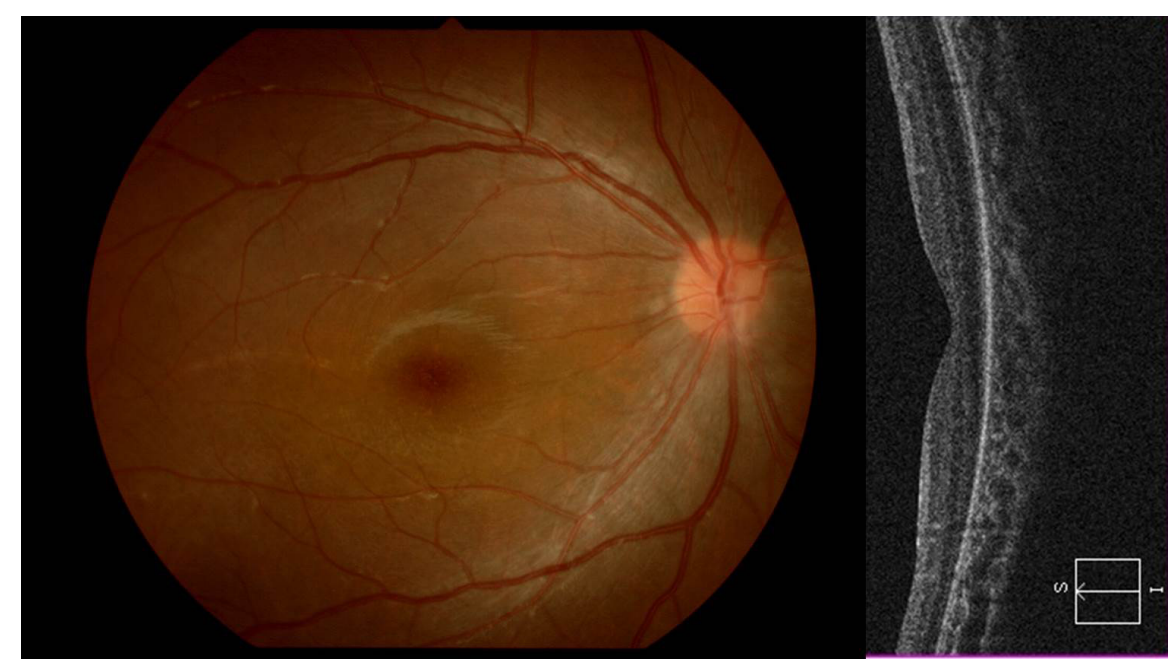

Figure I Preoperative right fundus and OCT imaging in Case I.

Notes: Peripheral retinal detachment with subretinal strands is visible. The macula appears uninvolved, but OCT shows the absence of the inner and outer segment junction line, indicating spontaneous attachment.

Abbreviation: OCT, optical coherence tomography. 


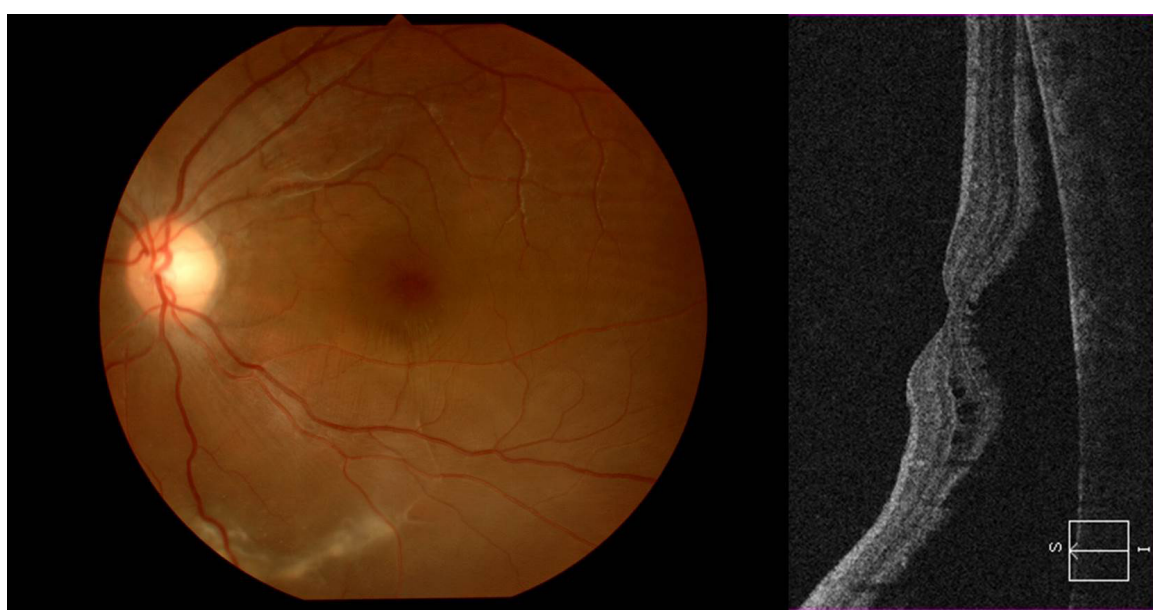

Figure 2 Preoperative left fundus and optical coherence tomography imaging in Case 2.

Note: Flat retinal detachment with subretinal strands and macular involvement are visible.

with myopia of -7.00 D in the left eye. Although slit-lamp examination found no abnormal findings in both eyes, fundus examination revealed flat retinal detachment involving the macula extending from 8 to 2 o'clock with two retinal holes and two lattice degenerations in the peripheral upper retina in the right eye, and lattice degeneration in the peripheral inferotemporal retina in the left eye.

\section{Surgical procedure}

After conjunctival peritomy, the four rectus muscles were isolated, and a 4-0 black silk suture was passed underneath each muscle for traction. Two sclerotomies with a 27 -gauge needle were placed at $4.0 \mathrm{~mm}$ behind the limbus, and 27-gauge twin chandelier illumination fibers (disposable Eckard TwinLight Chandelier; DORC International, Zuidland, the Netherlands) were inserted into the vitreous cavity on the side of the detached retina near the probable retinal break. A noncontact wide-angle viewing system (Resight ${ }^{\mathrm{TM}}$; Carl Zeiss Meditec,
Jena, Germany) (Cases 1 and 2) or a vitrectomy contact lens (HHV Dispo; Hoya, Tokyo, Japan) (Case 3) provided clear fundus views under the illumination inserted into the vitreous cavity (Figure 3 ). The retinal break could be identified and located with or without indentation of the sclera, and cryoretinopexy performed under the operating microscope. The illumination fiber was then removed, and the buckling procedure using silicone sponge (\#506; Labtician Ophthalmics, Oakville, ON, Canada), and if necessary, subretinal drainage through the external sclerotomy were performed. The illumination fiber could be inserted again to check the fundus. The 27-gauge sclerotomies were not sutured but were completely covered by conjunctiva at the end of surgery.

\section{Results}

Retinal reattachment was achieved in all eyes, and no complications occurred during follow-up periods of over 6 months. A small atrophic retinal hole at peripheral 9 o'clock was
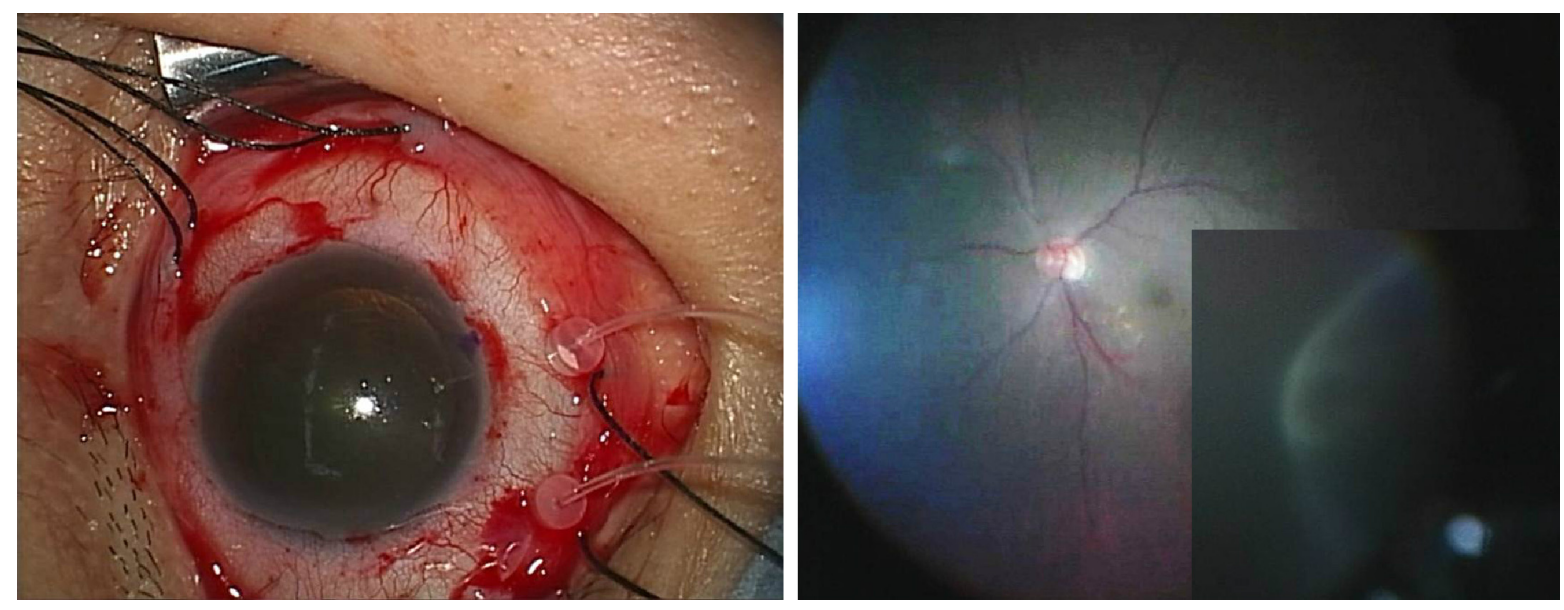

Figure 3 Image of surgical procedure.

Notes: Left: Intraoperative view of the inserted chandelier. Right: Intraoperative fundus image under chandelier illumination and the view of cryoretinopexy. 


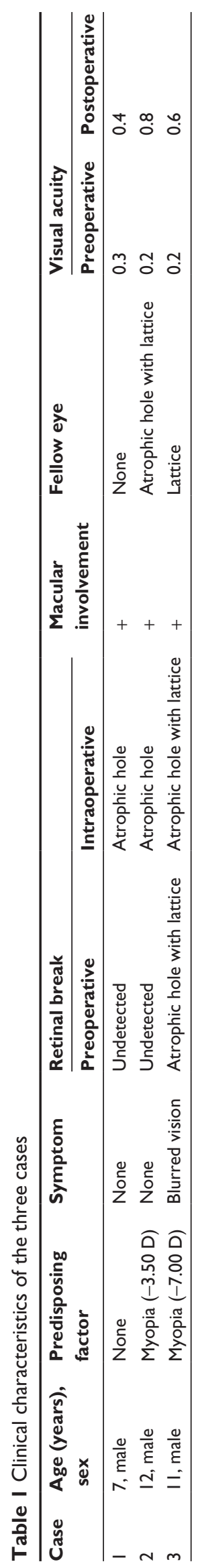

found in Case 1 and a similar retinal hole at 7 o'clock in Case 2 during surgery under chandelier illumination. Case 1 had unchanged postoperative visual acuity. Cases 2 and 3 achieved improved visual acuity of 0.8 and 0.6 , respectively. Table 1 summarizes the clinical features of our three cases.

\section{Discussion}

Recently, micro-incision vitrectomy surgery has become widely used and preferred as the first surgery for RRD. However, the scleral buckling procedure is still preferred for $\mathrm{RRD}$ in young patients. The scleral buckling procedure with chandelier illumination has some advantages in such cases. First, the entire surgical procedure can be performed under the surgical microscope without requiring the wearing of the binocular ophthalmoscope. Second, adjusting the viewing focus and magnification may be more helpful to identify preoperative undetected retinal breaks during surgery. Third, the image of the fundus can be shared during surgery, which is useful for surgical education. During the buckling procedure, the fundus is viewed through the indirect ophthalmoscope, which provides only a small inverted image, and only the operator can see the image without a teaching mirror. Experience is essential to obtain clear visualization of the fundus, especially in difficult cases involving small pupil, opaque media, or small hole. However, the use of chandelier illumination with the contact lens for vitrectomy or the wide-angle viewing system allows a clear view of the fundus through the operating microscope without extensive experience, and other medical staff can see the same view simultaneously. Such clear visualization facilitates surgery even in pediatric patients who often present with such difficulties.

Possible disadvantages of this method include light toxicity, lens damage, and infective endophthalmitis. However, light toxicity may be less likely because the chandelier illumination is placed far from the posterior retina, and lens damage can be avoided by careful procedures. We think that infectious endophthalmitis, although less common, is the most critical complication and is a serious concern. Checking for vitreous wick and complete removal if present are necessary, and the wound should be sutured. We think that small scleral wound, direct insertion through the sclera and not through the conjunctiva, and complete covering of the wound with conjunctiva are likely to reduce the risk of infection.

Previously, a pediatric case of retinal detachment with preoperatively undetected retinal tear was successfully treated by scleral buckling using 25 -gauge chandelier illumination combined with the wide-angle viewing system. ${ }^{12}$ Clear visualization helped to identify the preoperatively 
undetected retinal tear in our Cases 1 and 2. A series of 16 cases of retinal detachment were treated by scleral buckling using the noncontact wide-angle viewing system combined with 25 -gauge chandelier illumination. ${ }^{13}$ Vitrectomy was performed because of the development of PVR in three eyes and subretinal hemorrhage in two eyes.

The differences between our present and previously reported methods were the gauge of the chandelier and the use of the contact lens for vitrectomy, in addition to the wide-angle viewing system. Previous methods used a 25 -gauge chandelier, whereas we used a 27 -gauge chandelier. The smaller gauge chandelier needs only a small sclerotomy, and may decrease vitreous wicking from the sclerotomy site, resulting in reduced risk of infective endophthalmitis. The wide-angle viewing system is useful, especially in a patient with small pupil, but is not always necessary. The contact lens for vitrectomy is more widely used than the wide-angle viewing system, which can provide direct view of the fundus despite the restricted viewing angle, and combined with the scleral compression technique, it can allow viewing of the peripheral fundus.

All of our patients had macular lesion, but only Case 3 complained of visual disturbance. Moreover, no retinal break was identified preoperatively in Cases 1 and 2, but viewing under the microscope was very effective to find such defects. In Case 1, fundus examination showed macular attachment, but OCT showed the absence of the inner and outer segment junction line, suggesting chronic macular detachment followed by spontaneous attachment, indicating poor functional prognosis. Retinal detachment is not easy to detect, so all our cases were diagnosed with amblyopia or visual disturbance due to unknown cause. We emphasize the need to examine the peripheral fundus, which may show more prominent retinal detachment or subretinal strands, to detect possible retinal detachment as the cause of visual disturbance in pediatric patients. Moreover, OCT may help to reveal retinal detachment not detectable using the ophthalmoscope.

Our three pediatric patients with RRD were treated with the scleral buckling procedure with chandelier illumination under the wide-angle viewing system or contact lens for vitrectomy. All three patients had flat detachment involving the macula at initial presentation, which was reattached without complications. However, visual improvement was restricted in one patient. Chandelier illumination has potential disadvantages, such as the risks of infective endophthalmitis, lens injury, and light toxicity, but use in the buckling procedure for pediatric RRD has many advantages, especially in eyes with small or undetected retinal tear.

\section{Disclosure}

The authors report no conflicts of interest in this work.

\section{References}

1. Al-Zaaidi S, Al-Rashaed S, Al-Harthi E, Al-Kahtani E, Abu El-Asrar AM. Rhegmatogenous retinal detachment in children 16 years of age or younger. Clin Ophthalmol. 2013;7:1001-1014.

2. Fivgas GD, Capone A Jr. Pediatric rhegmatogenous retinal detachment. Retina. 2001;21(2):101-106.

3. Sadeh AD, Dotan G, Bracha R, Lazar M, Loewenstein A. Characteristics and outcomes of paediatric rhegmatogenous retinal detachment treated by segmental scleral buckling plus an encircling element. Eye (Lond). 2001;15(pt 1):31-33.

4. Oono Y, Uehara K, Haruta M, Yamakawa R. Characteristics and surgical outcomes of pediatric rhegmatogenous retinal detachment. Clin Ophthalmol. 2012;6:939-943.

5. Soheilian M, Ramezani A, Malihi M, et al. Clinical features and surgical outcomes of pediatric rhegmatogenous retinal detachment. Retina. 2009;29(4):545-551.

6. Gonzales CR, Singh S, Yu F, Kreiger AE, Gupta A, Schwartz SD. Pediatric rhegmatogenous retinal detachment: clinical features and surgical outcomes. Retina. 2008;28(6):847-852.

7. Chang PY, Yang CM, Yang $\mathrm{CH}$, et al. Clinical characteristics and surgical outcomes of pediatric rhegmatogenous retinal detachment in Taiwan. Am J Ophthalmol. 2005;139(6):1067-1072.

8. Yokoyama T, Kato T, Minamoto A, et al. Characteristics and surgical outcomes of paediatric retinal detachment. Eye (Lond). 2004;18(9):889-892.

9. Wang NK, Tsai CH, Chen YP, et al. Pediatric rhegmatogenous retinal detachment in East Asians. Ophthalmology. 2005;112(11):1890-1895.

10. Weinberg DV, Lyon AT, Greenwald MJ, Mets MB. Rhegmatogenous retinal detachments in children: risk factors and surgical outcomes. Ophthalmology. 2003;110(9):1708-1713.

11. Butler TKH, Kiel AW, Orr GM. Anatomical and visual outcome of retinal detachment surgery in children. Br J Ophthalmol. 2001;85(12): 1437-1439.

12. Kita M, Fujii Y, Kawagoe N, Hama S. Scleral buckling with a noncontact wide-angle viewing system in the management of retinal detachment with undetected retinal break: a case report. Clin Ophthalmol. 2013;7:587-589.

13. Aras C, Ucar D, Koytak A, Yetik H. Scleral buckling with a non-contact wide-angle viewing system. Ophthalmologica. 2012;227(2):107-110.
Clinical Ophthalmology

\section{Publish your work in this journal}

Clinical Ophthalmology is an international, peer-reviewed journal covering all subspecialties within ophthalmology. Key topics include: Optometry; Visual science; Pharmacology and drug therapy in eye diseases; Basic Sciences; Primary and Secondary eye care; Patient Safety and Quality of Care Improvements. This journal is indexed on Submit your manuscript here: http://www.dovepress.com/clinical-ophthalmology-journal

\section{Dovepress}

PubMed Central and CAS, and is the official journal of The Society of Clinical Ophthalmology (SCO). The manuscript management system is completely online and includes a very quick and fair peer-review system, which is all easy to use. Visit http://www.dovepress.com/ testimonials.php to read real quotes from published authors. 